\title{
Neoadjuvant nivolumab plus ipilimumab in resectable non-small cell lung cancer
}

\begin{abstract}
Joshua E Reuss (D) , , ${ }^{1,2}$ Valsamo Anagnostou, ${ }^{1,2}$ Tricia R Cottrell, ${ }^{1}$ Kellie N Smith, ${ }^{2}$ Franco Verde, ${ }^{3}$ Marianna Zahurak, ${ }^{1}$ Mara Lanis, ${ }^{1}$ Joseph C Murray, ${ }^{1,2}$ Hok Yee Chan, ${ }^{2}$ Caroline McCarthy, ${ }^{4}$ Daphne Wang, ${ }^{1,5}$ James R White, ${ }^{1}$ Stephen Yang, ${ }^{1,6}$ Richard Battafarano, ${ }^{1,6}$ Stephen Broderick, ${ }^{1,6}$ Errol Bush, ${ }^{1,6}$ Malcolm Brock, ${ }^{1,6}$ Jinny Ha, ${ }^{1,6}$ David Jones, ${ }^{7,8}$ Taha Merghoub, ${ }^{9,10}$ Janis Taube, ${ }^{1,5}$ Victor E Velculescu, ${ }^{1,2}$ Gary Rosner, ${ }^{1}$ Peter Illei, ${ }^{1,5}$ Drew M Pardoll, ${ }^{1,2}$ Suzanne Topalian, ${ }^{1,2}$ Jarushka Naidoo (D) , ${ }^{1,2}$ Ben Levy, ${ }^{1,2}$ Matthew D Hellmann, ${ }^{4,10}$ Julie R Brahmer, ${ }^{1,2}$ Jamie E Chaft, ${ }^{4,10}$ Patrick M Forde ${ }^{1,2}$
\end{abstract}

To cite: Reuss JE,

Anagnostou V, Cottrell TR, et al. Neoadjuvant nivolumab plus ipilimumab in resectable nonsmall cell lung cancer. Journal for ImmunoTherapy of Cancer 2020;8:e001282. doi:10.1136/ jitc-2020-001282

JEC and PMF contributed equally.

Accepted 05 August 2020

\section{ABSTRACT}

Background We conducted the first trial of neoadjuvant PD-1 blockade in resectable non-small cell lung cancer (NSCLC), finding nivolumab monotherapy to be safe and feasible with an encouraging rate of pathologic response. Building on these results, and promising data for nivolumab plus ipilimumab (anti-CTLA-4) in advanced NSCLC, we expanded our study to include an arm investigating neoadjuvant nivolumab plus ipilimumab.

Methods Patients with resectable stage IB $(\geq 4 \mathrm{~cm})-$ IIIA (American Joint Committee on Cancer Tumor Node Metastases seventh edition), histologically confirmed, treatment-naïve NSCLC received nivolumab $3 \mathrm{mg} / \mathrm{kg}$ intravenously plus ipilimumab $1 \mathrm{mg} / \mathrm{kg}$ intravenously 6 weeks prior to planned resection. Nivolumab $3 \mathrm{mg} / \mathrm{kg}$ was given again approximately 4 and 2 weeks preoperatively. Primary endpoints were safety and feasibility with a planned enrollment of 15 patients. Pathologic response was a key secondary endpoint.

Results While the treatment regimen was feasible per protocol, due to toxicity, the study arm was terminated early by investigator consensus after 9 of 15 patients were enrolled. All patients received every scheduled dose of therapy and were fit for planned surgery; however, 6 of $9(67 \%)$ experienced treatment-related adverse events (TRAEs) and $3(33 \%)$ experienced grade $\geq 3$ TRAEs. Three of 9 patients (33\%) had biopsy-confirmed tumor progression precluding definitive surgery. Of the 6 patients who underwent resection, 3 are alive and disease-free, 2 experienced recurrence and are actively receiving systemic treatment, and one died postoperatively due to acute respiratory distress syndrome. Two patients who underwent resection had tumor pathologic complete responses ( $p C R s)$ and continue to remain disease-free over 24 months since surgery. Pathologic response correlated with pre-treatment tumor PD-L1 expression, but not tumor mutation burden. Tumor KRAS/STK11 co-mutations were identified in 5 of 9 patients (59\%), of whom two with disease progression precluding surgery had tumor KRAS/ STK11/KEAP1 c0-mutations.
Conclusions Though treatment was feasible, due to toxicity the study arm was terminated early by investigator consensus. In light of this, and while the long-term disease-free status of patients who achieved pCR is encouraging, further investigation of neoadjuvant nivolumab plus ipilimumab in patients with resectable NSCLC requires the identification of predictive biomarkers that enrich for response.

\section{INTRODUCTION}

Despite curative-intent surgery, the majority of patients with resectable non-small cell lung cancer (NSCLC) relapse and die from their disease, ${ }^{12}$ highlighting a critical need for therapeutic innovation in this patient population. PD-1 pathway blockade has revolutionized the treatment of advanced NSCLC and is now the backbone of all FDA-approved first-line therapies for locally advanced or metastatic NSCLC lacking a targetable driver mutation. ${ }^{3}$

We reported the first trial of neoadjuvant PD-1 blockade in resectable NSCLC, finding nivolumab (anti-PD-1) to be safe and feasible with major pathologic response $(\leq 10 \%$ residual viable tumor in resected specimen) observed in 9 of 20 cases $(45 \%) .{ }^{4}$ Building on these results, and coupled with encouraging data for nivolumab plus ipilimumab (anti-CTLA-4) in advanced NSCLC, ${ }^{5} 6$ our study was amended to include an arm investigating neoadjuvant nivolumab plus ipilimumab (NCT02259621). Here, we report clinical safety and pathologic response data with genomic and molecular correlates from this study arm, which was terminated early by the consensus of the investigators due to unacceptable toxicity. 


\section{METHODS}

\section{Patient and public involvement}

Patient advocates from the Stand Up To Cancer and LUNGevity foundations worked together with study investigators to inform the design and execution of this trial.

\section{Patient selection and study design}

In this multicenter, open-label single-arm phase Ib/ II study (NCT02259621) conducted at Johns Hopkins University (JHU) and Memorial Sloan Kettering Cancer Center (MSKCC), adults $\geq 18$ years of age with resectable stage IB $(\geq 4 \mathrm{~cm})$-IIIA (American Joint Committee on Cancer Tumor Node Metastases seventh edition) treatment-naïve histologically confirmed NSCLC were eligible for enrollment. Other inclusion criteria included Eastern Cooperative Oncology Group performance status $0-1$, normal organ function and adequate pulmonary function for resection. Key exclusion criteria included active autoimmune disease, ongoing systemic steroids ( $>10 \mathrm{mg}$ daily prednisone equivalents) or other immunosuppressive therapy, active concurrent malignancy, history of symptomatic interstitial lung disease, preoperative chemotherapy, and any prior treatment with PD-1 or CTLA-4 pathway blockade.

Enrolled patients received nivolumab $3 \mathrm{mg} / \mathrm{kg}$ intravenously together with ipilimumab $1 \mathrm{mg} / \mathrm{kg}$ intravenously 6 weeks prior to planned resection. Two additional doses of nivolumab $3 \mathrm{mg} / \mathrm{kg}$ were given at approximately 4 and 2 weeks preoperatively (online supplementary figure 1 ). All patients were offered standard postoperative adjuvant chemotherapy \pm radiation as indicated. Primary endpoints were feasibility and safety, with feasibility defined as a delay in surgery of $\leq 24$ days from the preplanned surgery date and safety defined by adverse events according to common terminology criteria for adverse events (CTCAE) V.4.0. A six-patient run-in was performed to preliminarily assess safety. Assessment of pathologic response was a key exploratory efficacy endpoint. Planned enrollment was 15 patients.

\section{Study assessments}

All eligible patients underwent appropriate cancer staging prior to study enrollment including pathologic assessment of mediastinal lymph nodes (if indicated), in addition to baseline PET-CT and contrast-enhanced CT or MRI of the brain and chest. Mandatory pre-treatment primary tumor core biopsy was performed. Repeat imaging was obtained within 7 days before surgery to assess radiographic response to neoadjuvant therapy and reaffirm resectability. Post-neoadjuvant treatment radiographic response was determined using response evaluation criteria in solid tumors (RECIST) V.1.1. ${ }^{7}$ Of note, all RECIST-assessed response determinations were unconfirmed, as only one post-neoadjuvant treatment imaging assessment was made prior to surgical resection.

Resected primary tumors were assessed for residual viable tumor on routine $\mathrm{H} \& \mathrm{E}$-stained slides by attending pathologists at JHU and MSKCC, as previously described. ${ }^{48}$
Multiplex immunofluorescence (mIF) was performed for select patients to compare the tumor microenvironment of pre-treatment biopsy samples with post-treatment resected tumor tissue as described in online supplementary methods. Immunohistochemistry and mIF were performed for pre-treatment tumor PD-L1 evaluation.

Identification of somatic tumor genomic alterations for JHU patients was made using whole exome sequencing of tumor and matched normal samples as previously described $^{49}$ and outlined in online supplementary methods. For MSKCC patients, genomic alterations were identified by targeted next-generation sequencing (NGS) using the MSK-IMPACT assay as described previously. ${ }^{10}$ Tumor mutation burden (TMB) from exome and targeted-NGS analyses was normalized as described in online supplementary methods, with normalized TMB estimates used for statistical analyses.

\section{Statistical analysis}

Protocol-defined Bayesian stopping rules were originally employed to determine feasibility and safety as previously described ${ }^{4}$ and outlined in online supplementary methods. Treatment-related adverse events (TRAEs) were defined as adverse events (AEs) with possible or likely attribution to study drugs. Demographics and safety, as well as clinical, radiographic, pathologic and molecular response data were tabulated using descriptive statistics. Spearman's correlation was used to assess association of pathologic response with pre-treatment tumor PD-L1 expression and TMB, respectively. Reported $p$ values are two-sided with significance level set at 0.05. Statistical analyses were performed using R V.3.4.4.

\section{RESULTS}

\section{Safety and clinical data}

Between July 2017 and March 2018, nine patients were enrolled to the study arm. Baseline demographics of enrolled patients are outlined in table 1. All nine patients received every scheduled immune-checkpoint blockade (ICB) dose and were fit for planned surgery without treatment-related delays, meeting criteria for feasibility. No patient discontinued study treatment due to AEs; however six of nine patients $(67 \%)$ experienced TRAEs, and three (33\%) experienced grade $(G)$ $\geq 3$ TRAEs including acute respiratory distress syndrome (ARDS; grade 5), as well as pneumonitis, rash, pruritus and headache (all grade 3) (table 2, online supplementary figure 2). ARDS and possible pneumonitis were attributed as possibly related TRAEs; although given the timing of events, and in the setting of complicated surgery, it was felt these were more likely postoperative complications unrelated to study treatment. One patient (11\%) had a RECIST-assessed unconfirmed partial response, while four $(44 \%)$ had stable disease and four (44\%) had progressive disease on imaging assessment (figure 1, table 3, online supplementary figure 2). Of the four patients with RECIST-assessed progressive disease, 
Table 1 Baseline demographics

\begin{tabular}{lllllll}
\hline $\begin{array}{l}\text { Patient } \\
\text { number }\end{array}$ & Age (years) & Gender & Ethnicity & Smoking status (pack years) & Histology & Pre-treatment stage* \\
\hline 1 & 56 & Female & White & Former (3) & ADC & IB \\
2 & 50 & Male & White & Active (9) & ADC & IIA \\
3 & 78 & Male & White & Former (56) & ADC & IIB \\
4 & 69 & Male & White & Former (35) & ADC & IIIA \\
5 & 53 & Male & White & Former (80) & ADC & IIIA \\
6 & 70 & Male & White & Former (11) & ADC & IIIA \\
\hline 7 & 67 & Male & White & Former (45) & ADC & IIIA \\
\hline 8 & 48 & Female & White & Former (37) & ADC & IIIA \\
9 & 64 & Male & White & Former (80) & SCC & IIIA \\
\hline
\end{tabular}

*Staged according to AJCC TNM 7th edition.

ADC, adenocarcinoma; SCC, squamous cell carcinoma; AJCC TNM, American Joint Committee on Cancer Tumor Node Metastases.

one underwent resection and continues to be diseasefree while three had biopsy-proven tumor progression with distant metastases during neoadjuvant therapy that precluded definitive surgery or chemoradiation (figure 1, table 3). In total, six of nine patients (67\%) underwent definitive resection. As of data cut-off on December 15, 2019, among the six patients who underwent resection, three are alive and disease-free, two experienced recurrence and are actively receiving systemic treatment, and one died postoperatively due to ARDS (figure 1, online supplementary figure 2). While no protocol-defined

Table 2 Treatment-related adverse events (TRAEs) of possible or likely attribution to study therapies for enrolled population $(n=9)$

\begin{tabular}{lll}
\hline Toxicity & $\begin{array}{l}\text { Grade 1-2 } \\
\mathbf{n}(\%)\end{array}$ & $\begin{array}{l}\text { Grade 3-5 } \\
\mathbf{n}(\%)\end{array}$ \\
\hline Rash & $3(33)$ & $1(11)$ \\
Pruritus & $1(11)$ & $1(11)$ \\
\hline Fatigue & $2(22)$ & 0 \\
\hline ARDS & 0 & $1(11)^{\star} \dagger$ \\
\hline Headache & 0 & $1(11)$ \\
\hline Pneumonitis & 0 & $1(11)^{\star}$ \\
\hline Abdominal pain & $1(11)$ & 0 \\
\hline Arthralgia & $1(11)$ & 0 \\
\hline Diarrhea & $1(11)$ & 0 \\
\hline Fever & $1(11)$ & 0 \\
\hline Hypothyroidism & $1(11)$ & 0 \\
\hline Infusion reaction & $1(11)$ & 0 \\
Nausea & $1(11)$ & 0 \\
\hline Psoriasis & $1(11)$ & 0 \\
\hline
\end{tabular}

*Suspected to be more likely related to post-surgical complications but coded as "possibly related" TRAEs to be conservative. †Grade 5 TRAE; all other grade 3-5 TRAEs were grade 3. ARDS, acute respiratory distress syndrome; TRAE, treatmentrelated adverse event. dose-limiting TRAEs met the pre-determined stopping boundary for safety, due to substantial clinical toxicity, further study accrual was halted early by consensus of the investigators.

\section{Pathologic assessment and genomic analyses}

Pathologic, genomic and molecular data are summarized in figure 1, table 3 and online supplementary data. Pathologic complete response (pCR) was observed in two of six $(33 \%)$ resected tumors, both from patients with pretreatment stage IIIA disease. Pathologic response was significantly associated with pre-treatment tumor PD-L1 expression (Spearman rho $=-0.88 ; \mathrm{p}=0.02$ ) but not TMB (figure 1). Both patients with $\mathrm{pCR}$ had pre-treatment tumor PD-L1 expression $\geq 50 \%$ and continue to be diseasefree over 24 months since resection (figure 1, table 3). In patient 5 , who had partial radiographic response and PCR to neoadjuvant ICB, dense inflammatory infiltrates with abundant $\mathrm{CD}^{+}$cytotoxic $\mathrm{T}$ cells fully replaced tumor in the post-treatment resection tissue (figure 2A). In patient 4 , who had primary disease progression precluding resection, post-ICB treatment tumor biopsy revealed fully intact tumor with stromal macrophage-predominant infiltrates (figure $2 \mathrm{~B}$ ).

Co-occurring tumor mutations in KRAS/STK11 were identified in five of nine patients $(56 \%)$ including two (patients 2 and 4) with co-mutations in KRAS/STK11/ $K E A P 1$ who experienced primary tumor progression precluding definitive resection (figure 1 , table 3 ). A third patient (patient 8 ) with primary tumor progression precluding surgery had co-occurring mutations in BRAF/ STK11/TP53. All four patients with RECIST-assessed primary progression were TMB-low with low $(\leq 1 \%)$ pretreatment tumor PD-L1 expression (figure 1, table 3). Patient 1 with co-occurring KRAS/STK11 mutations and absent PD-L1 expression had progressive disease on presurgical imaging but underwent definitive surgery and had $100 \%$ residual tumor in the resected specimen. Interestingly, patient 5 , who achieved a pCR with neoadjuvant 
A
Pt. No. Residual
Clinical Course

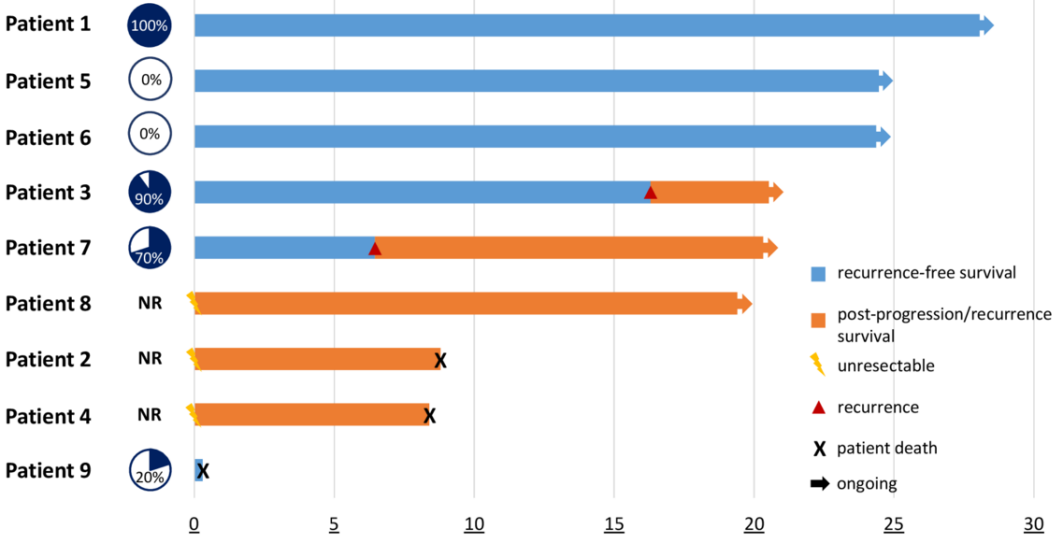

Months since surgery or biopsy-confirmed primary tumor progression

B

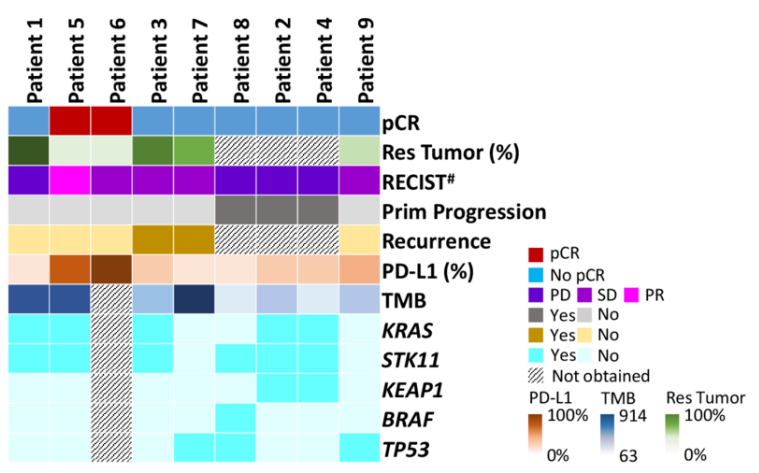

C

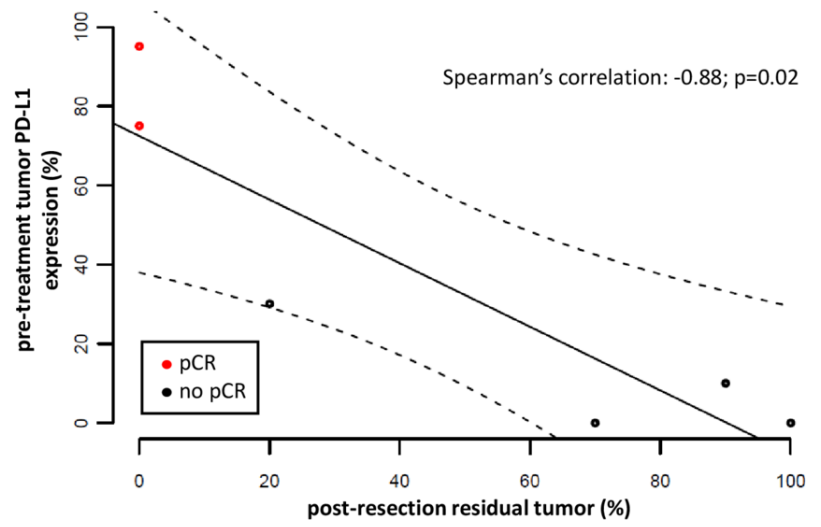

Figure 1 Clinical follow-up with radiographic and pathologic response characteristics plus molecular correlates. (A) Swimmerstyle clinical follow-up plot detailing clinical course of all enrolled patients. Residual viable tumor at resection is noted in the column to the right of patient number and to the left of outlined clinical course for that patient. NR indicates a tumor that was "not resected" due to primary disease progression precluding definitive surgery. Clinical course outlines time following surgery or biopsy-confirmed primary disease progression. (B) Genomic data for study patients including pathologic and radiographic response data, in addition to pre-treatment tumor PD-L1 expression. (C) Correlation between pre-treatment tumor PD-L1 expression and post-resection residual tumor. The solid dark line indicates the linear regression line, and the dashed lines indicate the upper and lower boundaries of the $95 \% \mathrm{Cl}$. ${ }^{\text {All }}$ radiographic RECIST assessments were unconfirmed, as only one post-neoadjuvant treatment imaging assessment was made prior to surgical resection. Patient 2 demonstrated clear progressive disease on PET imaging despite stable disease on RECIST response assessment of chest CT and was thus categorized as having "PD". NR, not resected; pCR, pathologic complete response; PD, progressive disease; PR, partial response; Prim. Progression, primary progression; RECIST, response evaluation criteria in solid tumors; Res. Tumor, residual tumor; SD, stable disease; TMB, tumor mutation burden.

ICB, had a tumor with co-occurring KRAS/STK11 mutations with high TMB and $75 \%$ pre-treatment tumor PD-L1 expression.

\section{DISCUSSION}

After demonstrating the safety and feasibility of neoadjuvant anti-PD-1 monotherapy in resectable NSCLC, ${ }^{4}$ we amended our study to include an arm investigating neoadjuvant nivolumab plus ipilimumab, a decision supported by promising data in metastatic NSCLC. ${ }^{56}$ While technically feasible without dose-limiting TRAEs meeting the pre-defined safety stopping rule, this regimen was associated with toxicity. Furthermore, when examining key metrics for perioperative clinical trials, in this study onethird of patients experienced primary disease progression precluding potentially curative surgery and a fourth patient died postoperatively. Given these factors, the study arm was closed to further accrual.

Grade 3 or higher TRAEs were observed in $33 \%$ of enrolled patients in our study, a numerically higher rate than that reported in an interim presentation of the NEOSTAR study, an ongoing phase II single-center study 
Table 3 Radiographic, pathologic and molecular response characteristics

\begin{tabular}{|c|c|c|c|c|c|}
\hline $\begin{array}{l}\text { Patient } \\
\text { number }\end{array}$ & $\begin{array}{l}\text { Radiographic } \\
\text { response* }^{\text {respons }}\end{array}$ & $\begin{array}{l}\text { Residual tumor } \\
(\%)\end{array}$ & $\begin{array}{l}\text { Pre-treatment } \\
\text { PD-L1 (\%) }\end{array}$ & $\begin{array}{l}\text { Normalized tumor } \\
\text { mutation burden }\end{array}$ & $\begin{array}{l}\text { Driver genes with sequence } \\
\text { alterations }\end{array}$ \\
\hline 1 & PD & 100 & 0 & 344 & KRAS, STK11 \\
\hline 3 & SD & 90 & 10 & 147 & KRAS, STK11, TP53 \\
\hline 4 & PD & $\mathrm{N} / \mathrm{A}$ & 1 & 63 & KRAS, KEAP1, STK11 \\
\hline 7 & $\mathrm{SD}$ & 70 & 0 & 914 & TP53 \\
\hline 8 & PD & $\mathrm{N} / \mathrm{A}$ & 0 & 78 & BRAF, STK11, TP53 \\
\hline 9 & $\mathrm{SD}$ & 20 & 30 & 99 & TP53 \\
\hline
\end{tabular}

*All radiographic RECIST assessments were unconfirmed, as only one post-neoadjuvant treatment imaging assessment was made prior to surgical resection.

†Clear metastatic disease on PET imaging despite stable disease on RECIST response assessment of chest CT and thus coded as having "PD".

‡Pre-treatment tumor tissue for patient 6 was insufficient for whole exome sequencing and thus genomic assessments could not be performed.

N/A, not applicable; pCR, pathologic complete response; PD, progressive disease; PR, partial response; RECIST, response evaluation criteria in solid tumors; SD, stable disease.

which randomized 44 patients with resectable NSCLC to neoadjuvant nivolumab or nivolumab plus ipilimumab. ${ }^{11}$ Differences in toxicity rates are likely attributable to sample size and overall the observed rate of $\geq$ G3 TRAEs in our study was similar to that seen with nivolumab plus ipilimumab in advanced NSCLC ${ }^{6}$ and in neoadjuvant studies of resectable melanoma. ${ }^{12}$ Nevertheless, the toxicity in our study arm exceeded that reported in neoadjuvant studies of anti-PD1 monotherapy ${ }^{411} 1314$ and combination ICB plus chemotherapy, ${ }^{15} 16$ which was a major determinant in our decision to terminate this study arm early.

All patients with imaging-assessed progressive disease in our study appeared to have true progression (vs pseudoprogression), as progressive disease was biopsy-confirmed in the three patients who were no longer surgical candidates, and $100 \%$ residual tumor was present in the resection specimen from the patient who underwent definitive surgery. Biopsy confirmation of progressive disease is important as, while not seen in this small study, cases of pseudoprogression have been observed in other studies of neoadjuvant checkpoint blockade, ${ }^{11}$ and it is vital that such patients are not excluded from potentially curative surgery. With the caveat of sample size, the rate of disease progression precluding surgery $(33 \%)$ observed in this study was greater than that seen in our prior neoadjuvant nivolumab monotherapy study $(5 \%) .{ }^{4}$ While neoadjuvant treatment was delivered over a greater length of time in our study of nivolumab plus ipilimumab (6 vs 4 weeks), time alone was unlikely the sole factor contributing to the higher observed rate of disease progression, as only $5 \%$ of patients randomized to the nivolumab plus ipilimumab arm of NEOSTAR (identical treatment duration) experienced progressive disease precluding surgery. ${ }^{11}$
However, early progression as a potential limitation of neoadjuvant ICB does have a corollary in advanced lung cancer; progressive disease as best response was observed in 23\% of patients with treatment-naïve advanced NSCLC randomized to the nivolumab plus ipilimumab arm $(\mathrm{n}=583)$ of the phase III CheckMate 227 trial. $^{6}$ Furthermore, the median time to response to nivolumab plus ipilimumab in this trial was 2.7 months, suggesting the unconfirmed response rate of $11 \%$ observed in our study may underestimate the true response rate with this regimen, though speculation on this is severely limited by sample size. For comparison, neoadjuvant chemotherapy has demonstrated a radiographic response rate of $\sim 50 \%$ in phase III trials in resectable NSCLC with only $9 \%$ of patients unable to undergo surgery. ${ }^{17} 18$ Taken together, this highlights the magnified importance of response rate, disease control and maintaining resectability in assessing the clinical utility of neoadjuvant ICB in resectable NSCLC, where surgery remains paramount to the goal of cure.

One possible explanation for the poor clinical outcomes observed in our study was the high incidence $(56 \%)$ of genomic alterations reported to be associated with ICB resistance. Co-mutations in KRAS/STK11 and KRAS/KEAP1 have both been associated with ICB resistance and poor survival in advanced NSCLC, ${ }^{19}{ }^{20}$ as have concurrent STK11/KEAP1 mutations, which frequently co-occur with KRAS mutations. ${ }^{21}{ }^{22}$ Furthermore, STK11/ KEAP1 co-mutations have been observed to occur twice as frequently in metastatic versus resectable lung adenocarcinoma, suggesting this molecular profile may be an indicator of aggressiveness. ${ }^{22}$ While limited by sample size, our finding of tumor progression precluding resection in patients with KRAS/STK11/KEAP1 co-mutations 
A Patient 5

CT Imaging

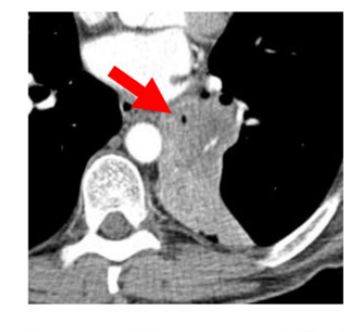

Pre-treatment

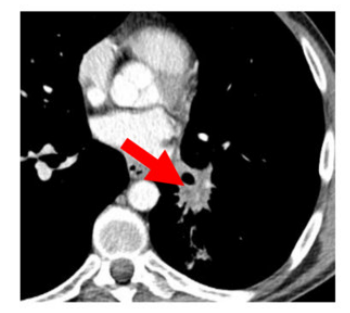

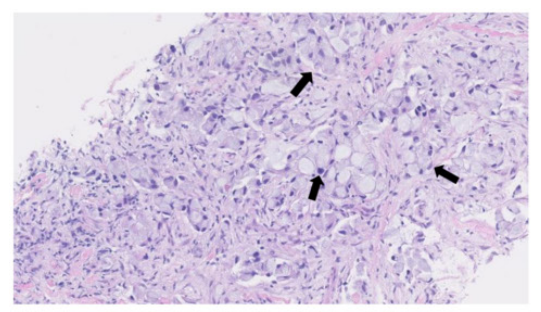

H\&E

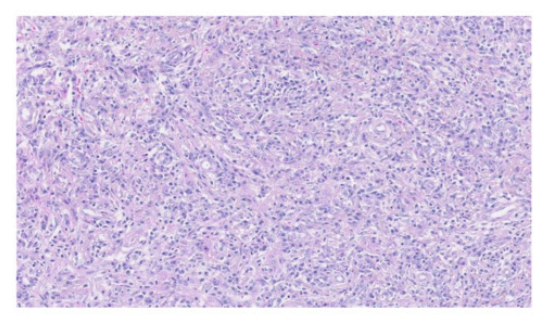

mIF

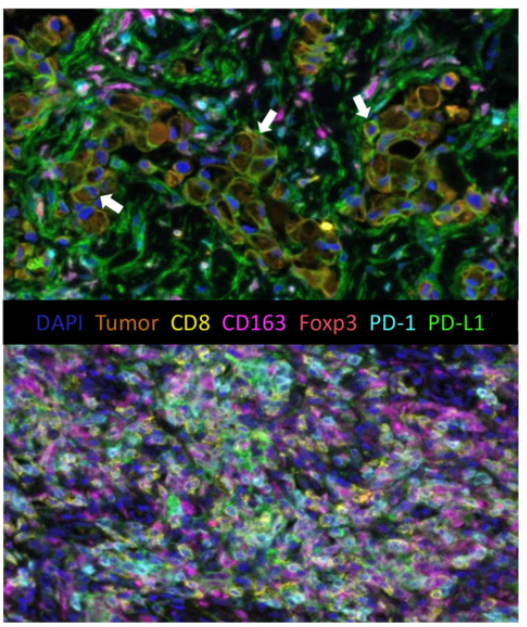

B

\section{Patient 4}

Pre-treatment
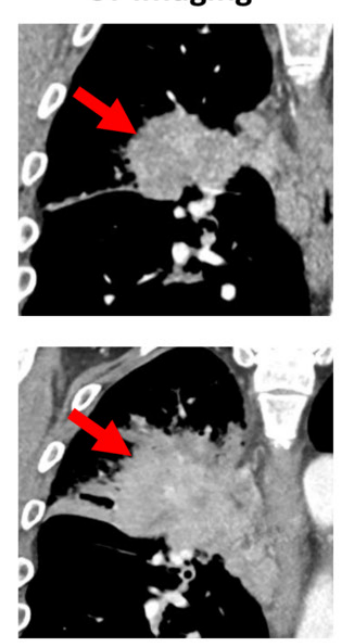

$H \& E$
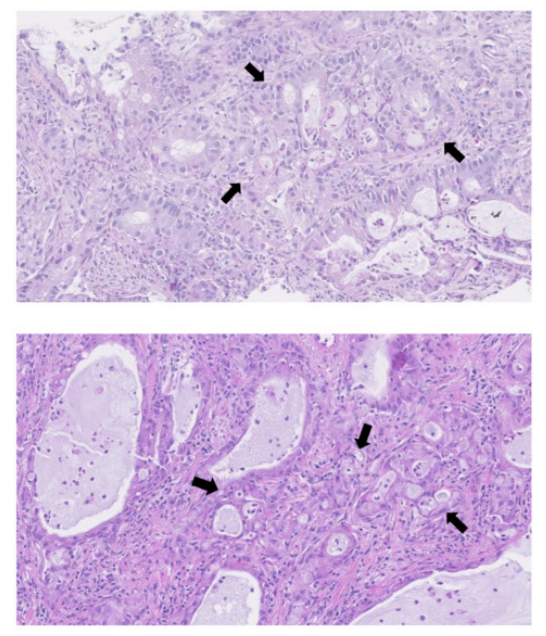

mIF

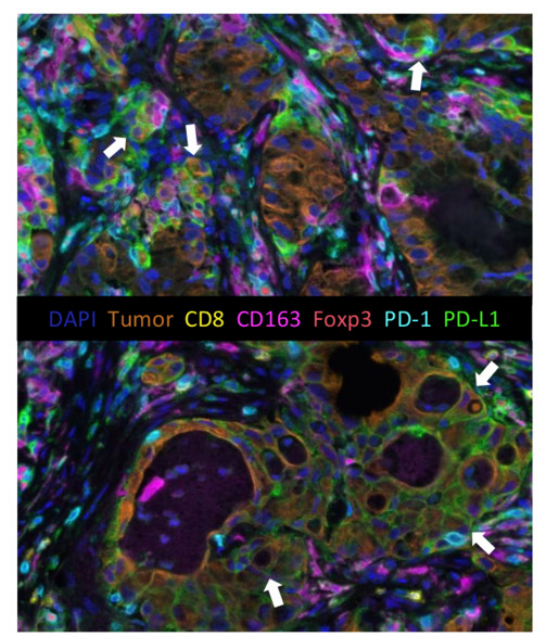

Figure 2 Radiographic and pathologic response to neoadjuvant nivolumab plus ipilimumab for (A) patient with pathologic complete response ( $\mathrm{pCR}$ ) and $(\mathrm{B})$ no pathologic response to treatment. (A) Radiographic and pathologic response for patient 5 with $\mathrm{pCR}$ to neoadjuvant nivolumab plus ipilimumab. Pre-treatment contrast-enhanced CT imaging demonstrates a $4.4 \times 4.2 \mathrm{~cm}$ left lower infrahilar mass (red arrow) encasing adjacent bronchi with posterior post-obstruction atelectasis. Pre-treatment biopsy demonstrates abundant infiltrating malignant signet ring cells distinguished by atypical eccentric nuclei surrounding a large mucinous vacuole (black arrows). The pre-treatment tumor shows abundant PD-L1 positive tumor and stromal cells (PD-L1 in green, cytokeratin expression in orange highlights tumor cells (white arrows)). Post-treatment pre-resection imaging demonstrates decreased size of mass now measuring $2.2 \times 2.7 \mathrm{~cm}$ (red arrow) with re-expansion of previously collapsed lung. Post-treatment resection tissue shows abundant inflammatory cells, cellular fibrosis and neovascularization; features typical of immune-mediated tumor regression. Multiplex immunofluorescence (mIF) highlights abundant cytotoxic T cells (CD8, yellow) and macrophages (CD163, magenta), as well as scattered regulatory T cells (Foxp3, red). No residual tumor cells are identified (note the absence of orange tumor cells on mIF), consistent with pCR. (B) Radiographic and histologic findings for patient 4 with primary tumor progression preventing definitive surgery. Pre-treatment contrast-enhanced CT imaging demonstrates a $6.8 \times 6.5 \mathrm{~cm}$ posterior right upper lobe mass (red arrow) encasing the right upper lobe bronchus. Posttreatment imaging demonstrates enlargement of mass to $8.3 \times 6.9 \mathrm{~cm}$ (red arrow) with worsening encasement and enlarging paratracheal adenopathy. On assessment of pre-treatment and post-treatment biopsies, atypical glandular structures (black arrows), including confluent (cribriform) glands, are present with no histologic evidence of tumor regression in post-treatment specimen. PD-L1 expression (green) is seen on tumor cells (orange, white arrows) both pre-treatment and post-treatment. Scant pre-treatment and post-treatment inflammatory infiltrates are composed largely of macrophages (CD163, magenta), which are predominantly localized to the intra-tumoral stroma. H\&E photomicrographs taken at $\times 200$ original magnification. mIF images taken at $\times 400$ original magnification. mIF, multiplex immunofluorescence. 
further supports this. That said, the predicted functional consequence of mutational profiles alone is likely insufficient to predict ICB response. In our study, patient 5 with tumor KRAS/STK11 co-mutations had a pCR to neoadjuvant ICB and continues to be disease-free. This tumor was TMB-high with high PD-L1 expression. Furthermore, in a retrospective analysis of the KEYNOTE-042 study, both STK11 and KEAP1 mutational status were not associated with survival or response to anti-PD1 therapy in advanced NSCLC. ${ }^{23}$

One additional consideration is whether patients with primary disease progression in our study had an aggressive disease phenotype resistant to ICB or hyperprogression induced by ICB. Hyperprogressive disease (HPD) is a putative response pattern to ICB that has yet to be fully elucidated or uniformly defined. Given this, speculation as to the occurrence of HPD in this small study is difficult. As all patients in our study with distant progression on ICB had pre-treatment node-positive disease, the presence of occult metastases in the setting of an underlying molecular predisposition for ICB resistance may have contributed to widespread progressive disease. Together, this suggests multiple intrinsic and extrinsic variables factor into ICB response, and underscores the need for further research to define reproducible predictive biomarkers.

Pre-treatment tumor PD-L1 expression, but not TMB, correlated with pathologic response in our study, the reverse of what was observed in our neoadjuvant PD-1 monotherapy study. ${ }^{4}$ Small sample size likely contributed to this difference, though pre-treatment PD-L1 expression was also found to correlate with pathologic response in the NEOSTAR study. ${ }^{11}$ With the caveat of sample size, both NEOSTAR and our study report a pCR rate of $\sim 30 \%$ with neoadjuvant nivolumab plus ipilimumab. ${ }^{11}$ In addition, neoadjuvant PD-1 blockade plus chemotherapy has demonstrated an encouraging pCR rate of $33 \%-59 \%$ with tolerable safety profile in both published and ongoing phase II trials in resectable NSCLC. ${ }^{16}{ }^{24}$ This is substantially higher than the median pCR rate observed with neoadjuvant chemotherapy, which has been reported at $\sim 4 \%$ in resectable NSCLC. ${ }^{25}$ With additional long-term follow-up and the pending completion of several phase III trials of neoadjuvant chemoimmunotherapy in resectable NSCLC, data will soon be available to evaluate the utility of pCR as a surrogate biomarker for survival in neoadjuvant ICB NSCLC trials, which will be vital in determining the clinical utility of this approach in resectable NSCLC.

In summary, though the treatment regimen was feasible, toxicity together with several cases of primary tumor progression precluding definitive resection led to early termination of our study. In light of this, and while the long-term disease-free status of patients who achieved pCR is encouraging, further investigation of neoadjuvant nivolumab plus ipilimumab in patients with resectable NSCLC requires the identification of predictive biomarkers that enrich for response.

\section{Author affiliations}

${ }^{1}$ Department of Oncology, Sidney Kimmel Comprehensive Cancer Center, Johns Hopkins, Baltimore, Maryland, USA

${ }^{2}$ The Bloomberg-Kimmel Institute for Cancer Immunotherapy, Johns Hopkins University, Baltimore, Maryland, USA

${ }^{3}$ Department of Radiology, Johns Hopkins, Baltimore, Maryland, USA

${ }^{4}$ Thoracic Oncology Service, Memorial Sloan Kettering Cancer Center, New York, New York, USA

${ }^{5}$ Department of Pathology, Johns Hopkins, Baltimore, Maryland, USA

${ }^{6}$ Department of Surgery, Johns Hopkins, Baltimore, Maryland, USA

${ }^{7}$ Thoracic Surgery Service, Memorial Sloan Kettering Cancer Center, New York, New York, USA

${ }^{8}$ Druckenmiller Center for Lung Cancer Research, Memorial Sloan Kettering Cancer Center, New York, New York, USA

${ }^{9}$ Parker Institute for Cancer Immunotherapy, Memorial Sloan Kettering Cancer Center, New York, New York, USA

${ }^{10}$ Weill Cornell Medical College, New York, New York, USA

Correction notice This article has been corrected since it was published online. The author name 'Matthew Hellmann' was updated to 'Matthew D Hellmann'.

Twitter Joshua E Reuss @Joshua_Reuss and Patrick M Forde @FordePatrick

AcknowledgmentsWe would like to thank the patients and families who participated in this study, as well as the research teams at Johns Hopkins University and Memorial Sloan-Kettering Cancer Center.

Contributors Conceptualization and study design: VA, KNS, MZ, GR, JEC, PMF. Data acquisition and analysis: all authors. Data interpretation: all authors. Original drafting of manuscript and figures: JER. Manuscript revision and editing: all authors. All authors read and approved the final manuscript, and have agreed to be accountable for all aspects of the work.

Funding This work was funded in part by grants from the National Institutes of Health (CA121113, CA006973, CA180950, CA233259, T32 CA193145, T32 CA009071-38, R01 CA142779), Stand Up To Cancer - CRI Immunology Dream Team (SU2C-AACR-DT1012), Stand Up To Cancer - American Cancer Society Lung Cancer Dream Team, Bristol-Myers Squibb International Immuno-Oncology Network, Conquer Cancer: The ASCO Foundation - Young Investigator Award (90083528), LUNGevity Foundation, the V Foundation, Lung Cancer Foundation of America, MacMillan Foundation, Eastern Cooperative Oncology Group-American College of Radiology Imaging Network, Swim Across America, The Allegheny Health Network - Johns Hopkins Research Fund and the Maryland Department of Health and Mental Hygiene Cigarette Restitution Fund Program, the Commonwealth Foundation, the Bloomberg-Kimmel Institute for Cancer Immunotherapy, Memorial Sloan Kettering Cancer Center (P30 CA008748), Johns Hopkins University Cancer Center (P30 CA006973).

Disclaimer Funding sources had no role in the study design, data collection/ analysis/interpretation, report writing or decision to submit the article for publication.

Competing interests JER has received grants from Conquer Cancer: The ASCO Foundation. VA has received grants from the National Institutes of Health, The LUNGevity Foundation, The V Foundation, Swim Across America, The Allegheny Health Network - Johns Hopkins Research Fund and the Maryland Department of Health and Mental Hygiene Cigarette Restitution Fund Program, Bristol-Myers Squibb, and Personal Genomic Diagnostics. KS has received personal fees from Illumina. JRW is the founder and owner of Resphera Biosciences LLC. SB has received personal fees from Bristol-Myers Squibb. DJ has been on the advisory boards of Diffusion Pharmaceuticals, Merck, and AstraZeneca. TM has received grants from Bristol-Myers Squibb, Surface Oncology, Kyn Therapeutics, Infinity Pharmaceuticals, Peregrine Pharmaceuticals, Adaptive BioTechnologies, Leap Therapeutics, and Aprea; has received personal fees from Leap Therapeutics, Immunos Therapeutics, and Pfizer; is co-founder and holds equity in Imvaq Therapeutics; and has filed patents for work related to PD-1 and CTLA-4. JT has received grants from Akoya Biosciences and Bristol-Myers Squibb, has received non-financial support from Akoya Biosciences, and has been on the advisory boards of Bristol-Myers Squibb, Merck, AstraZeneca, Compugen, and Regeneron. VEV has received grants from the National Institutes of Health, The Commonwealth Foundation, and Bloomberg-Kimmel Institute for Cancer Immunotherapy; has been on the advisory boards of Bristol-Myers Squibb, Genentech, Merck, and Takeda Pharmaceuticals; and is the founder, serves on board of directors and owns stock in Delfi Diagnostics and Personal Genome Diagnostics. GR has received personal fees from Medicago and owns stock in Johnson \& Johnson. 
PI has received grants from Bristol-Myers Squibb and Biomarker Strategies, LLC; has received personal fees from AbbVie, AstraZeneca, Bayer, Veran Medical Technologies Inc., and Roche Diagnostics; and has been on the advisory boards of AstraZeneca and Guardant Health. DMP and ST have received research grants from Bristol-Myers Squibb and Compugen; have received personal fees from Aduro Biotech, Five Prime Therapeutics, Tizona Therapeutics, DNAtrix, RAPT, WindMIL, Dragonfly Therapeutics, Ervaxx, Amgen, Medlmmune, Merck, Compugen, Dynavax, Immunomic Therapeutics, Janssen Oncology, Immunocore, BristolMyers Squibb, Arbor Pharmaceuticals, and NexImmune; and own equity in Aduro Biotech, Potenza Therapeutics, Five Prime Therapeutics, Tizona Therapeutics, DNAtrix, RAPT, WindMIL, Dragonfly Therapeutics, Ervaxx, and Trieza Therapeutics. $\mathrm{JN}$ has received grants from AstraZeneca and Merck; has been on the advisory boards of AstraZeneca, Bristol Myers-Squibb, and Genentech/Roche; and has received personal fees from AstraZeneca, Bristol-Myers Squibb, and Merck. BL has received grants from Eli Lilly, Genentech, Bristol-Myers Squibb, AstraZeneca, Turning Point Therapeutics, and Amgen; and has received personal fees from Eli Lilly, Genentech, AstraZeneca, Celgene, Pfizer, Merck, Novartis, and Takeda. MH has received grants from Bristol-Myers Squibb; has received personal fees from Bristol-Myers Squibb, Merck, AstraZeneca, Genentech/Roche, Nektar, Syndax, Mirati, Shattuck Labs, Immunai, Blueprint Medicines, Achilles, Arcus, and Eli Lilly; owns equity in Shattuck Labs, Immunai, and Arcus; and has filed patents related to the use of tumor mutation burden to predict response to immunotherapy. JRB has received grants from AstraZeneca, Bristol-Myers Squibb, Genentech/Roche, Merck, RAPT Therapeutics, Inc., and Revolution Medicines; has received personal fees from Genentech/Roche; has been on the advisory boards of Amgen, Bristol-Myers Squibb, Genentech/Roche, Eli Lilly, GlaxoSmithKline, Merck, and Sanofi; and has been on the Data and Safety Monitory boards of GlaxoSmithKline and Sanofi. JEC has received grants from the National Institutes of Health; and has also received grants and personal fees from Merck, Bristol-Myers Squibb, Genentech, and AstraZeneca. PMF has received grants from the LUNGevity Foundation, Stand Up To Cancer, AstraZeneca, Bristol-Myers Squibb, Kyowa, Novartis, and Corvus; and has been on the advisory boards of Abbvie, AstraZeneca, and Bristol-Myers Squibb. The remaining authors declare no conflicts of interest.

\section{Patient consent for publication Not required.}

Ethics approval Patients provided written informed consent for inclusion. The study protocol was approved by the institutional review boards of JHU and MSKCC.

Provenance and peer review Not commissioned; externally peer reviewed.

Data availability statement Data are available on reasonable request. Patient and correlative datasets beyond those published in supplementary data are available from the corresponding author on reasonable request.

Open access This is an open access article distributed in accordance with the Creative Commons Attribution Non Commercial (CC BY-NC 4.0) license, which permits others to distribute, remix, adapt, build upon this work non-commercially, and license their derivative works on different terms, provided the original work is properly cited, appropriate credit is given, any changes made indicated, and the use is non-commercial. See http://creativecommons.org/licenses/by-nc/4.0/.

\section{ORCID iDs}

Joshua E Reuss http://orcid.org/0000-0002-6401-9080

Jarushka Naidoo http://orcid.org/0000-0002-3470-8686

\section{REFERENCES}

1 Goldstraw P, Chansky K, Crowley J, et al. The IASLC Lung Cancer Staging Project: proposals for revision of the TNM stage groupings in the forthcoming (eighth) edition of the TNM Classification for Lung Cancer. J Thorac Oncol 2016;11:39-51.

2 Higgins KA, Chino JP, Berry M, et al. Local failure in resected N1 lung cancer: implications for adjuvant therapy. Int $J$ Radiat Oncol Biol Phys 2012;83:727-33.

3 Remon J, Passiglia F, Ahn MJ, et al. Immune checkpoint inhibitors in thoracic malignancies: review of the existing evidence by an IASLC expert panel and recommendations. J Thorac Oncol 2020.

4 Forde PM, Chaft JE, Smith KN, et al. Neoadjuvant PD-1 blockade in resectable lung cancer. N Engl J Med 2018;378:1976-86.

5 Hellmann MD, Ciuleanu T-E, Pluzanski A, et al. Nivolumab plus ipilimumab in lung cancer with a high tumor mutational burden. $N$ Engl J Med 2018;378:2093-104.
6 Hellmann MD, Paz-Ares L, Bernabe Caro R, et al. Nivolumab plus ipilimumab in advanced non-small-cell lung cancer. N Engl J Med 2019;381:2020-31

7 Eisenhauer EA, Therasse P, Bogaerts J, et al. New response evaluation criteria in solid tumours: revised RECIST guideline (version 1.1). Eur J Cancer 2009;45:228-47.

8 Cottrell TR, Thompson ED, Forde PM, et al. Pathologic features of response to neoadjuvant anti-PD-1 in resected non-small-cell lung carcinoma: a proposal for quantitative immune-related pathologic response criteria (irPRC). Ann Onc 2018;29:1853-60.

9 Anagnostou V, Niknafs N, Marrone K, et al. Multimodal genomic features predict outcome of immune checkpoint blockade in nonsmall-cell lung cancer. Nature Cancer 2020;1:99-111.

10 Cheng DT, Mitchell TN, Zehir A, et al. Memorial Sloan KetteringIntegrated Mutation Profiling of Actionable Cancer Targets (MSKIMPACT): a hybridization capture-based next-generation sequencing clinical assay for solid tumor molecular oncology. J Mol Diagn 2015;17:251-64.

11 Cascone T, William WN, Weissferdt A, et al. Neoadjuvant nivolumab (N) or nivolumab plus ipilimumab (Ni) for resectable non-small cell lung cancer (NSCLC): clinical and correlative results from the NEOSTAR study. JCO 2019;37:8504.

12 Rozeman EA, Menzies AM, van Akkooi ACJ, et al. Identification of the optimal combination dosing schedule of neoadjuvant ipilimumab plus nivolumab in macroscopic stage III melanoma (OpACIN-neo): a multicentre, phase 2, randomised, controlled trial. Lancet Oncol 2019;20:948-60.

13 Kwiatkowski DJ, Rusch VW, Chaft JE, et al. Neoadjuvant atezolizumab in resectable non-small cell lung cancer (NSCLC): interim analysis and biomarker data from a multicenter study (LCMC3). JCO 2019;37:8503.

14 Gao S, Li N, Gao S, et al. Neoadjuvant PD-1 inhibitor (Sintilimab) in NSCLC. J Thorac Oncol 2020;15:816-26.

15 Provencio M, Nadal E, Insa A, et al. Neoadjuvant chemoimmunotherapy for the treatment of stage IIIA resectable non-smallcell lung cancer (NSCLC): a phase II multicenter exploratory studyfinal data of patients who underwent surgical assessment. JCO 2019;37:8509.

16 Shu CA, Gainor JF, Awad MM, et al. Neoadjuvant atezolizumab and chemotherapy in patients with resectable non-small-cell lung cancer: an open-label, multicentre, single-arm, phase 2 trial. Lancet Oncol 2020;21:786-95.

17 Gilligan D, Nicolson M, Smith I, et al. Preoperative chemotherapy in patients with resectable non-small cell lung cancer: results of the MRC LU22/NVALT 2/EORTC 08012 multicentre randomised trial and update of systematic review. Lancet 2007;369:1929-37.

18 Felip E, Rosell R, Maestre JA, et al. Preoperative chemotherapy plus surgery versus surgery plus adjuvant chemotherapy versus surgery alone in early-stage non-small-cell lung cancer. J Clin Oncol 2010;28:3138-45.

19 Arbour KC, Jordan E, Kim HR, et al. Effects of co-occurring genomic alterations on outcomes in patients with KRAS-mutant non-small cell lung cancer. Clin Cancer Res 2018;24:334-40.

20 Skoulidis F, Goldberg ME, Greenawalt DM, et al. STK11/LKB1 mutations and PD-1 inhibitor resistance in KRAS-mutant lung adenocarcinoma. Cancer Discov 2018;8:822-35.

21 Skoulidis F, Byers LA, Diao L, et al. Co-occurring genomic alterations define major subsets of KRAS-mutant lung adenocarcinoma with distinct biology, immune profiles, and therapeutic vulnerabilities. Cancer Discov 2015;5:860-77.

22 Shen R, Martin A, Ni A, et al. Harnessing clinical sequencing data for survival stratification of patients with metastatic lung adenocarcinomas. JCO Precis Oncol 2019;3:9.

23 Cho BC LG, Kowalski DM, Kasahara K, et al. CT084-Relationship between STK11 and KEAP1 mutational status and efficacy in KEYNOTE-042: pembrolizumab monotherapy versus platinum-based chemotherapy as first-line therapy for PD-L1-positive advanced NSCLC. 2020 AACR Virtual Annual Meeting I 2020.

24 Provencio M, Nadal E, Insa A, et al. OA13.05 NADIM study: updated clinical research and outcomes. J Thorac Oncol 2019;14:S241.

25 Hellmann MD, Chaft JE, William WN, et al. Pathological response after neoadjuvant chemotherapy in resectable non-small-cell lung cancers: proposal for the use of major pathological response as a surrogate endpoint. Lancet Oncol 2014;15:e42-50. 